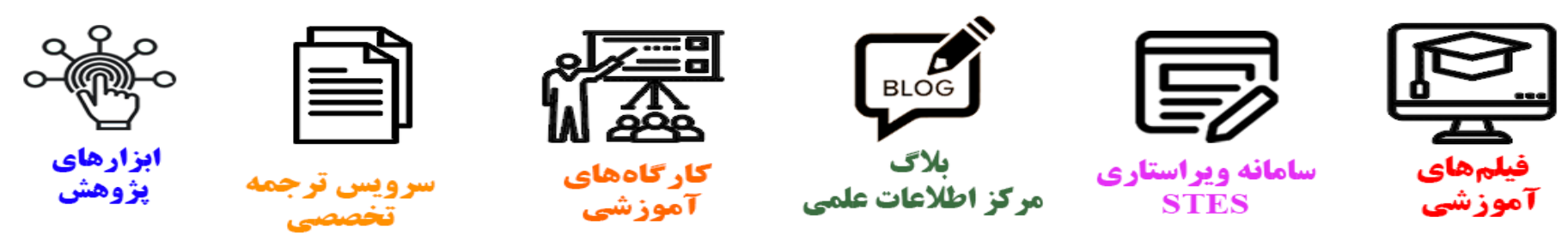

\title{
(c)
}

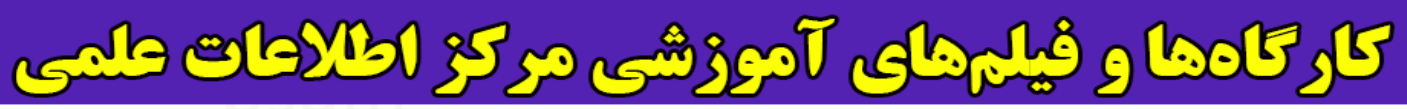
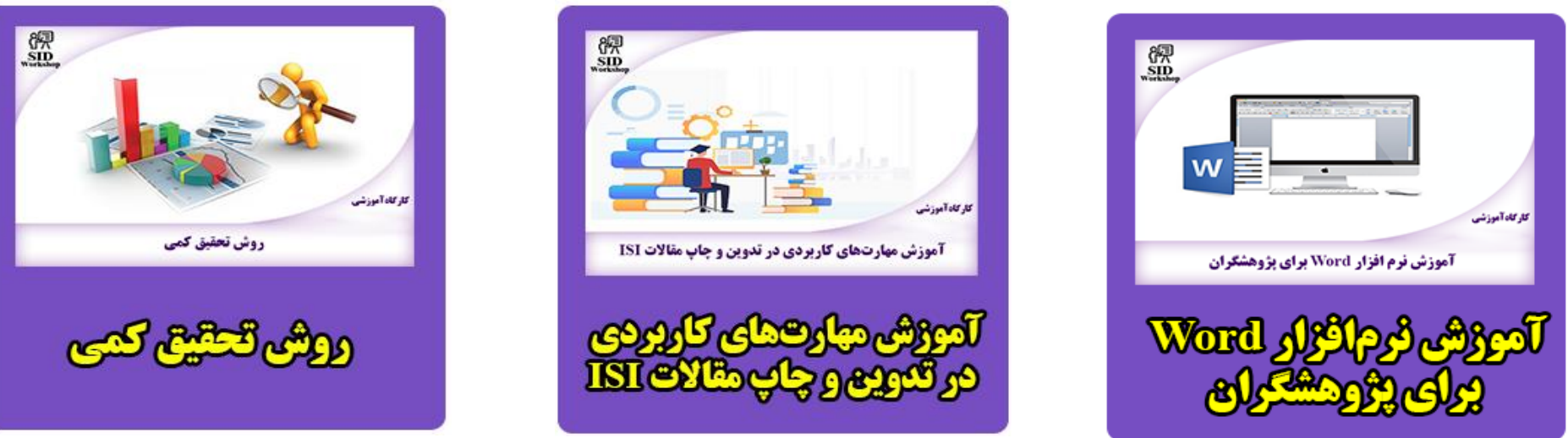


\title{
International Journal of Engineering \\ RESEARCH

Journal Hom epage: www.ije.ir

\section{Analysis of Packet Loss and Latency Control for Robust IPTV over Mobile WiMAX and LTE Assessment}

\author{
M. Akram ${ }^{a}{ }^{*}$, F. Zafar ${ }^{b}$ \\ a Department of Information Technology, University of Central Punjab, Lahore, Pakistan \\ ${ }^{b}$ Department of Computer Science, Government College University, Lahore, Pakistan
}

\section{PAPER INFO}

\section{Paper history:}

Received 7 February 2012

Received in revised form 5 September 2012

Accepted 15 November 2012

\section{A $B \begin{array}{lllllll} & S & T & R & A & C & T\end{array}$}

There are different schemes for streamed audio video (AV) IPTV content across mobile WiMAX to reduce packet loss and latency. The objective of this paper is to verify the effectiveness of forward error correction (FEC) techniques and to suggest the techniques for robustness problems and to analyze the issues either due to AV coding encoding or due to access network. The paper concentrates on handoff and handover delay, mobile WiMAX downlink burst mapping and the reliability of IPTV over LTE system.

\section{Keywords:}

IPTV

WiMAX

LTE

IPv6

MPEG

FEC

Coding

Streaming

Signal Brust

doi: 10.5829 /idosi.ije.2013.26.03c.02

\begin{tabular}{|c|c|c|c|}
\hline \multicolumn{4}{|c|}{ NOMENCLATURE } \\
\hline $\mathrm{S}$ & Slice composed of original bursts & WS & The width of stripe \\
\hline в & Burst & $\neq \omega$ & The number of wasted aditional or extra slots in stripe allocation \\
\hline bí & Original bursts & $\mathrm{Q}$ & The number of network elements \\
\hline sí & Slots, time slice & \multirow{2}{*}{\multicolumn{2}{|c|}{ Greek Symbols }} \\
\hline $\mathrm{n}$ & Number of burst & & \\
\hline $\mathrm{H}$ & The height of the fram & $\mathrm{O}$ & The complexity of algorithm \\
\hline$\hat{\mathrm{w}} \mathrm{S}$ & Temperary width of slice & $\sum$ & Summation sum over ... sum from \\
\hline hí & The temporary height of each burst & $\varepsilon$ & Element of \\
\hline đ & Delay processing, propagation, queuing & $\forall$ & All of, for any, for each \\
\hline ẅí & Adaptive width of each burst & $\ulcorner\cdot\lrcorner$ & Rounds to closest integger \\
\hline
\end{tabular}

\section{INTRODUCTION}

Internet Protocol Television (IPTV) provides digital information and audio video contents using high speed internet [1]. This IP based network is managed to

*Corresponding Author Email: engineerakram@ucp.edu.pk (M. Akram) provide quality of service (QoS), quality of experience (QoE), security, reliability and interactivity. Before transmission, the IPTV contents must be digitized as MPEG format. IPTV has an intelligent content management to deliver the programs by encoded video stream, application layer coding schemes and retransmission of extra redundant data. There are several services of IPTV like interactivity (two way 
communication between consumers and service providers), downloading, electronic program guide, personal video recorder, time shifted TV and video on demand (VOD). It has high-speed access channels via set-top boxes or receiving equipment. The IPTV setup for fixed and mobile subscribers is shown in Figure 1. Service Provider Core/Edge IP Network is the core network of the service provider and includes hardware. The access network connects the service provider to the subscriber's home as a fixed subscriber of IPTV via set top box. The IP based core network is connected with mobile WiMAX for mobile subscribers.

In IPTV over mobile WiMAX setup the AV content rate adapter is used for adapting the IPTV service bandwidth.This adapter is used for available WiMAX bandwidth and the IP encapsulation.

The two primary protocols used for IPTV are internet group multicast protocol (IGMP) version 2 for channel change signalling for live $\mathrm{TV}$ and real time streaming protocol ( RTSP) for video on demand. Figure $2 \mathrm{a}$ shows the serialised AV signal for IPTV transmitting through DVB-H. Figure $2 \mathrm{~b}$ shows the receiving end of IPTV.

The protocol stack to transmit the broadcasting content for IPTV services is shown in Figure 3a. IPTV sends MPEG type of AV contents in sequence to network side after RTP, UDP and layer 2 encapsulated. Figure $3 \mathrm{~b}$ shows a protocol stack in each entity to manage the channel registration and release to provide IPTV service [2]. IPTV head-end captures audio video and then $\mathrm{AV}$ is processed by encoding each individual channel into a digital video format MPEG-2-4 before being sent over the IP network.

The AV encoded streams performance is inherently a function of available link bandwidth and delay characteristics. So, analysis of performance including packet loss, latency, jitter and minimum peak throughput is discussed to quantify IPTV over the core mobile WiMAX infrastructure.

To reconstruct streamed video across an IEEE 802.16E mobile WiMAX channel, the different schemes are discussed in this paper. The paper concentrates on the redundant data to verify the effectiveness of forward error correction (FEC) bit rate and appropriate compression rate. To analyse the tradeoff between FEC and degradation of video quality for given packet loss ratio, the encoders are used as test sequences for the channel error models.

For IPTV services the mobile WiMAX is discussed as a medium which has handover latency components same as in mobile IPv6 and latency jitter in mobile WiMAX which is the cause of delay in sensitive applications. There are three latency components of mobile IPv6.

There are different distribution technologies for IPTV network like delivery over fibre access network, ADSL network, next generation cable TV network, internet, mobile WiMAX and over LTE. This paper concentrates on IPTV delivery over mobile WiMAX. The IPTV transmission system over LTE has also some issues as reliability, packet loss rate and delay time. The cellular networks like WiMAX and LTE are not only for conventional voice service but also for data service, for the readability of services the different techniques are required to bring new generation on the mobile market.

This study is based on following sections; related work will be discussed in section 2, proposed system will be described in section 3 , comparison and performance evolution will be described in section 4 and conclusion will be discussed in section 5 .

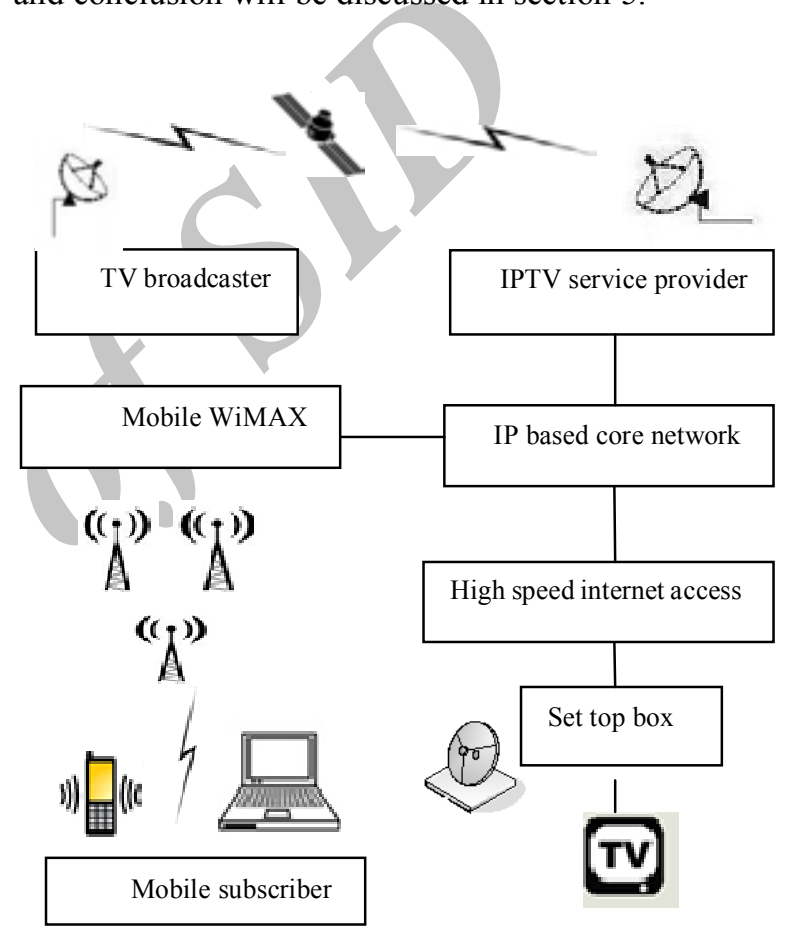

Figure 1. IPTV setup for fixed and mobile subscribers

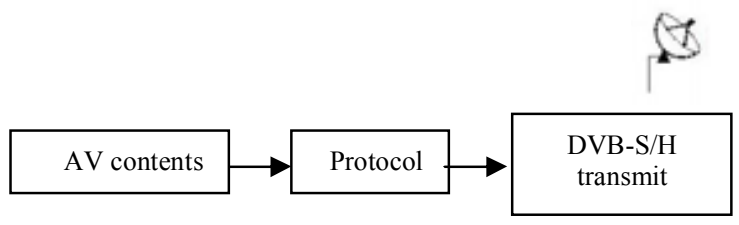

Figure 2a. Transmitting AV signals

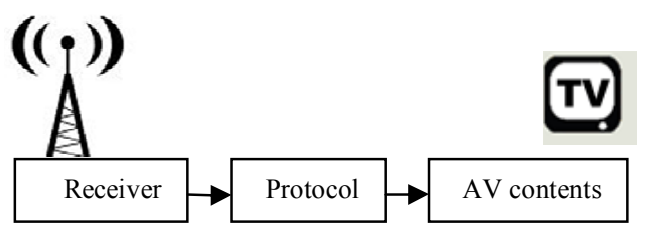

Figure 2b. Receving AV signals 


\begin{tabular}{|c|c|c|}
\hline AV content & & AV content \\
\hline MPEG & & MPEG \\
\hline RTP & & RTP \\
\hline UDP & & UDP \\
\hline IP & & IP \\
\hline Ethernet & & Ethernet \\
\hline Physical layer & Physical layer & Physical layer \\
\hline IPTV head-end & First hop last hop router & IP set top \\
\hline
\end{tabular}

Figure 3a. Protocol stack to transmit broadcasting contents for IPTV

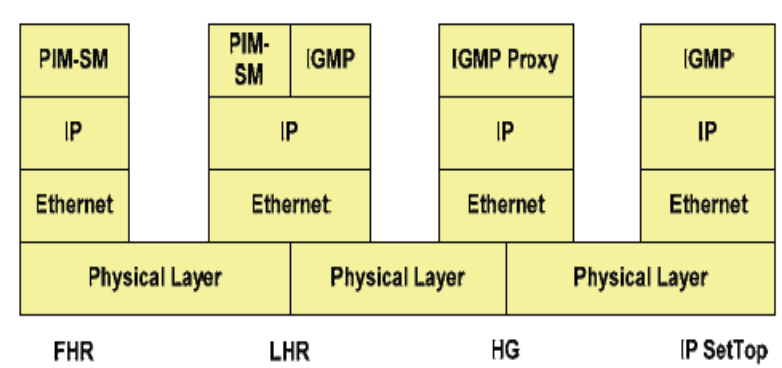

Figure 3b. Protocol stack to manage the multicast group for IPTV [2]

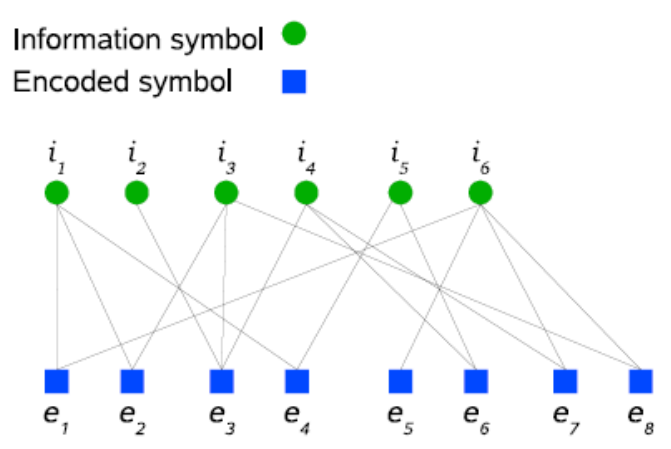

Figure 4. Encoding graph of an LT code [3]

\section{RELATED WORK}

\section{1. Attenuation of Packet Loss and Noisy}

Channel This section refers to accessing the collection of previously published and unpublished information and proposes techniques to attenuate or reduce the packet loss and noisy channel. The short introduction of these techniques is given.
2. 1. 1. Fountain Codes Fountain codes are generated from a given set of source symbols which refer to the fact that these codes do not exhibit a fixed code rate. These codes are used in such network where files are transmitted in multiple small packets, each of which is either received without error or not received [4]. Standard file-transfer protocols simply chop a file up into $\mathrm{K}$ packet-sized pieces, and then repeatedly transmit each packet until it is successfully received. A back-channel is required for the transmitter to find out which packets need retransmittance. In contrast, fountain codes make packets that are random functions of the whole file.

The transmitter sprays packets at the receiver without any knowledge that which packets are received. Once the receiver has received any $\mathrm{N}$ packets, where $\mathrm{N}$ is just slightly greater than the original file-size $\mathrm{K}$, the whole file can be recovered. In summary, the number of packets required to have certain probability. The expected encoding cost per packet is $\mathrm{K}=2$ packet operations, since on average half of the packets must be added up. While a random code is not in the technical sense; a "perfect" code for the erasure channel (it has only a chance of 0.289 of recovering the file when $\mathrm{K}$ packets have arrived), is nearly perfect.

A drawback of the fountain concept is the limitation to download-and-play services that is this concept is not directly applicable for real-time service like video streaming.

2. 1. 2. Luby Transform Codes Luby Transform (LT) code is a sparse random linear fountain code, with a super-cheap approximate decoding algorithm. The LT code retains the good performance of the random linear fountain code, while considerably reducing the encoding and decoding complexities. In LT the H.264/MPEG-4 AVC video compression standard is used to provide significantly better objective video quality results than two state of the art techniques in applications where a high video quality is desired [3]

Figure 4 describes encoding graph of an LT code. Eight encoded symbols are generated from $\mathrm{k}=6$ information symbols. The degree of an encoded symbol is the number of information symbols that were used to generate it. For example, the degree of el is equal to two. The LT decoder tries to recover the original information symbols from the received encoded symbols. To decrease the bit error rate (BER) of LT codes by virtually increasing the number of information symbols, the unequal error protection properties are used.

The input symbols are not necessarily reproduced among the output symbols therefore the LT codes are not systematic, which does not guarantee a high probability of decode ability from any subset of received output symbols. 
2. 1. 3. Intrarefrsh Macroblocks The insertion of intra-refresh (IR) and macroblocks (MB) are encoded in to pictures through motion compensated prediction which allows temporal error propagation to be arrested if matching in a previous picture.

In the H.264/AVC JM implementation, various IR schemes exist such as random, which sets a maximum percentage of MBs, or cyclic, which replaces each line of the picture in turn in cyclic order. Notice that naturally encoded IR MBs are also inserted into predicatively coded p-pictures when inter coding brings limited or no advantage. (e.g., this may occur during rapid motion or when a new object that is not present in a previous picture is uncovered) [5].

2. 1. 4. Adaptive Scheme Application layer, adaptive rateless channel coding scheme is used to reconstruct streamed video across an IEEE 802.16e (mobile WiMAX) channel, rateless code decoding is reliant upon the identification of clean symbols. In the adaptive scheme [6], the probability of channel loss (PL) serves to predict the amount of redundant data to be added to the payload. Assume that "bursty" error conditions are generated by the widely used GilbertElliott model, which is a form of hidden Markov model with a good and bad state.

In the Gilbert-Elliott model $p g b$ is probability of the transition from the good state to the bad state, and $p b g$ is the probability of transition from the bad state to the good state. Then pgg and $p b b$ are the probabilities of staying in the good state and the bad state respectively as shown in Figure 5.

This latter function is performed by PHY-layer FEC which passes up correctly received blocks of data (through a cyclic redundancy check) but suppresses erroneous data. For example, in IEEE 802.16e [7], a binary, nonrecursive convolution encoder with a constraint length and a native rate operates at the physical layer.

The scheme reduces the FEC overhead. Whichever scheme reduces, the number of corrupted packets reduces the overall delay introduced into the video stream. However, if packets are simply not received then the adaptive rateless coding scheme cannot help. The main disadvantage of using model generated loss traces is that statistical properties may not fit to the statistical properties of a measured trace as they are likely to be biased by model limitations.

2. 2. Reducing Handoff Handover Latency Handoff and handover have become a crucial factor in quality of service responsive networks. In WiMAX the handoff latency ratio is based on its hierarchical model and it is required to use centralized control approach to reduce end to end packet delay. For this purpose, previously proposed techniques and studies are given.

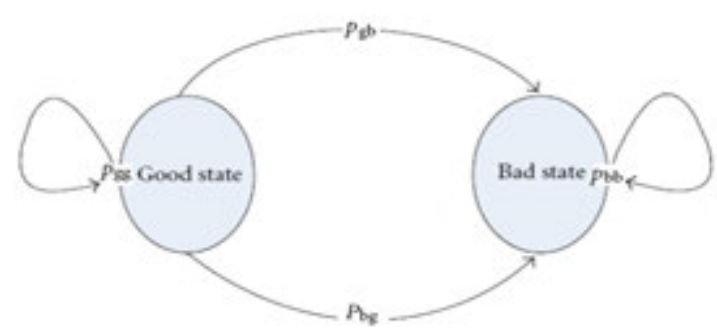

Figure 5. Gilbert-Elliott two-state model

2. 2. 1. A Cross Layer Approach To reduce delay, jitter, and packet loss innovative cross-layer content, delivery architecture is capable of receiving information from the network and adaptively tune transport parameters, bit rates, and QoS mechanisms according to the underlying network conditions [8]. The proposed adaptive cross layer video streaming system is composed of a video server and a video client. The server streams the audiovisual object to the client via an IP Diff-Serv network using the RTP protocol. The client decodes and composes the original MPEG-4 scene. When network congestion occurs, less important AV streams are dropped automatically by the active queue implemented in the Diff-Serv router. The IP Diff-Serv marker tags each video data packet belonging to one AV object with one of the supported Diff-Serv class of service to reflect object priority. Hence, important objects will be marked with a low drop precedence to guarantee a minimum loss, and so on. Object datapackets within the same class are then transmitted over the selected transport layer with the corresponding bearer capability. The disadvantage of AV object-based methods is that all packets of objects are given the same importance regardless of distortion caused by each one. The major drawback of existing cross-layer designs is that the simplification occurs at the system modelling phase rather than the problem solving phase.

2. 2. 2. WiMAX with MIPv6 Handover Mobile IPV6 is an important factor to study the latency components. There are three latency components. The first one is D1 (delay 1) which is the time it takes at link-layer to switch to the new access medium. The second one is D2 which is the time to detect the new IPv6 network, complete DAD (duplicate address detection) and configure the new CoA (care of address). The third latency component is D3 which is finally the delay to update the home agent and all other communication and parameters.

IPv6 provides real-implementation results for significant parts of the handover process through measurements in a real MIPv6 implementation on a wireless test bed based on IEEE 802.11b [9]. IPv6 is the networking technology of choice to enable the internet 
working and unification of these diverse technologies in the effort to move to an all-IP 4G environment. Mobility is seen as an integral part of future networks, where the initiatives for next generation networks (NGN) will meet more traditional and established networks to form heterogeneous architectures. The existing link layer (L2) handoff schemes in technologies such as IEEE 802.11, 3GPP, and 3GPP2 do not consider the network layer (L3) handoff. In addition, the L3 handoff like Mobile IPv6 does not consider the L2 handoff. Although the HMIPv6 and FMIPv6 protocols and their extensions can reduce the L3 handoff latency, they cannot reduce the $\mathrm{L} 2$ handoff latency. The handover management of IPv6 faces some problems. When a mobile node moves from one access point to another access point in a small coverage area (micro-mobility), the IPv6 reduces frequent handover. MIPv6 generates significant amount of signalling traffic in the core network, even for local movement, followed by long interruption during the handover. The network mobility basic support protocol (NEMO BSP) [10] is based on MIPv6. It inherits the drawbacks of MIPv6 such as long signaling delay and movement detection time. Moreover, the delay caused by the movement of the mobile router (MR) affects all mobile nodes $(\mathrm{MN})$ in the mobile network. Another problem is that this protocol does not cover mobile nodes movement which moves in or out of a mobile network. It means that mobile nodes should have mobility protocol for own mobility.

2. 2. 3. Node Mobility Supporting Scheme For MIPv6 Networks A node mobility supporting scheme is proposed for MIPv6 networks. This scheme introduces mobile access gateway (MAG) functions to the MR and extends Proxy MIPv6 (PMIPv6) network to a mobile network [11]. Therefore, MN handovers between the mobile network and PMIPv6 network with only IPv6 stack. PMIPv6 protocol and NEMO without any modifications for local mobility anchor (LMA) and MAG is implemented. The results shows link layer handover latency is critical to total handover latency. Also, effective handover shows that default router validation (DRI) results in poor quality in interactive application.

But the seamlessness during handover is not supported by PMIPv6 and the wireless link between MR and mobile access gateway (MAG) carries collective traffic for mobile network, therefore a mechanism to guarantee the seamlessness during handover may be required.

\section{PROPOSED TECHNIQUES}

To reduce the noise and packet loss in IPTV (based on physical layer protocol stack), the proposed technique is a process in which an extra burst is added after the original burst with taking data from one protocol and translating it into another protocol by using FEC. The IPTV has the MPEG transport stream with DVB-H. To transmit IP data over DVB networks, the multi-protocol encapsulation method (MPE) is used. MPE is a data link layer protocol which provides the functional and procedural means to transfer data and to detect errors that may occur in the physical layer. To understand the proposed technique, the technical features of DVB-H and implementation of proposal is given.

3. 1. Time Slicing, MPE-FEC and FEC If the data are continuously transmitted as in terrestrial DVB, the time slicing is used in DVB-H to transmit data in periodic bursts with higher instantaneous bit rates [12]. Figure 6a shows time slicing.

Depending on the transmission bit rate, burst size and burst duration, the off time $\Delta t$ in the transmission stream can vary. The burst parameters are shown in Figure $6 \mathrm{~b}$. The DVB-H receiver can use this off time to synchronize and initialize soft hand over another cell that would be impossible without use of time slicing.

The time difference is indicated by each burst to the next burst. Users do not notice a discontinuous transmission due to information received in last burst.To encapsulate multiple IP streams into the MPEG DVB transport stream multi-protocol encapsulation (MPE) is used. MPE forward error correction (MPE-FEC) is used to improve the robustness of the system for mobile users. MPE-FEC scheme consists of a Reed-Solomon (RS) code in conjunction with a virtual block interweaver. Figure 7a shows the MPE-FEC frame with application data table [13].

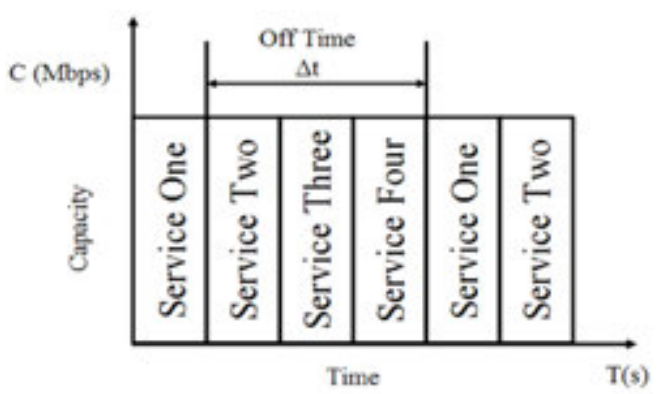

Figure 6a.Time slicing illustration [12]

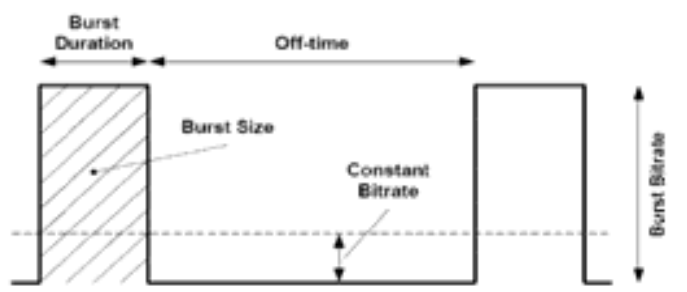

Figure 6b. Time slicing burst parameters [12] 


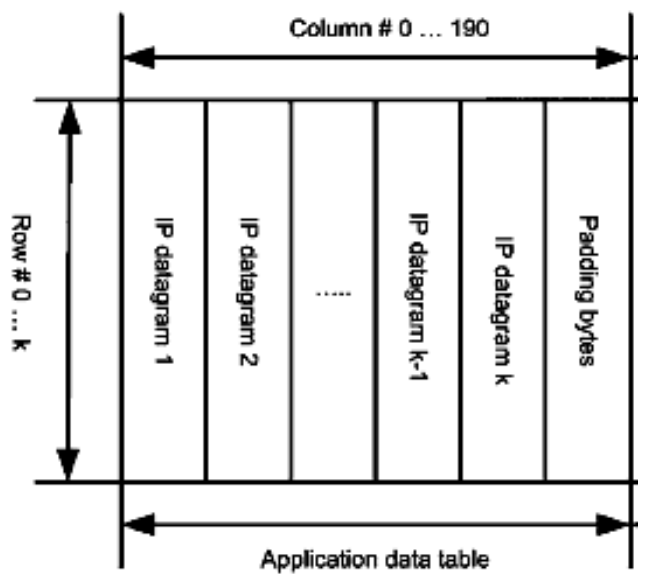

Figure 7a. MPE-FEC frame with application data table [13]

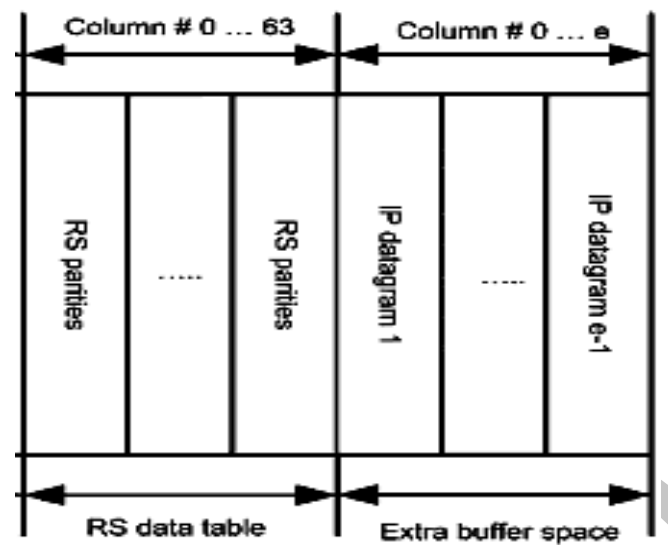

Figure 7b. MPE-FEC frame with extra buffer space

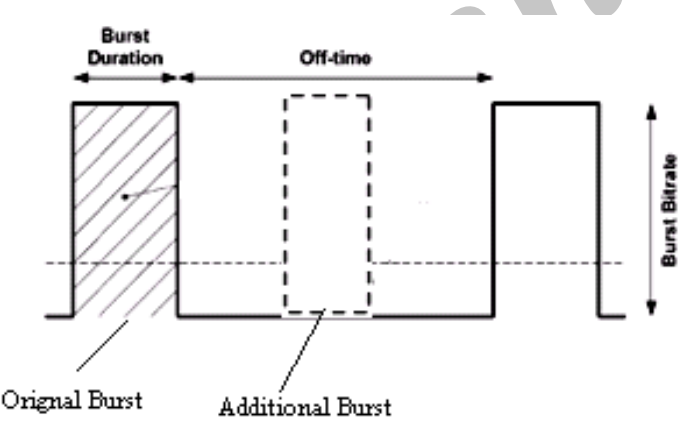

Figure 8. Example of additional burst

Figure $7 \mathrm{~b}$ shows MPE-FEC frame with extra buffer space. The two parts of MPE-FEC are application data table (ADT) with IP data and RS data table (RSDT) with RS parity data. By applying a RS code on each row the RSDT is computed after filling the ADT columnwise. The proportion of transmitted columns in each table is a resulting coding rate. DVB-H standard allows padding to make the code stronger and perforating to make the code weaker. The use of MPE-FEC is always a trade-off between consumption of network capacity and robustness [14]. Application Layer Forward Error Correction (AL-FEC) is used to provide reliability in mobile broadcast systems. The Raptor codes adopted by DVB-H for file download services are systematic codes which are efficient implementation of fountain codes that operate at the application layer. To generate additional RTP streams, the FEC packets are used by the receiver to reconstruct lost packets.

There are two disadvantages for FEC techniques as:

- Additional bandwidth is needed to transmit FEC packets

- At the transmitter and the receiver stage the additional latency is introduced by FEC operations

3. 2. Proposed Technique Stategy This paper proposes the addition of an extra burst after the original burst with taking data from one protocol and translating it into another protocol using FEC. The extra burst containing parity data is transmitted several seconds after the informative burst. The informative burst indicates the initial time of next burst with new information and it indicates the time of the additional burst. Now, the coding (AL-FEC) rate for both burst depends on the size of the extra burst with parity data. The time of extra burst can be calculated with the sum of informative burst duration and off-time as shown in Figure 8.

With the addition of an extra burst, the AV content can be decoded in three different ways. At the receiving end the original informative burst can be received correctly by neglecting the additional one. Another condition is possible that there is only part of the original burst received at receiving end and receiver will need a part or all the additional burst. Here is another case that when the receiver receives only the additional burst but cannot receive original burst then additional burst help as a redundant data to recover the information transmitted. The original burst and the additional burst has a statistical relationship which resolves the robustness, loss with respect to distance and time. The recovery or the improvement of the data is based on the speed and the time between both bursts. The correctly reception of bursts can be calculated with the average carrier to noise ratio (CNR), standard deviation of shadow fading in $\mathrm{dB}$ and distance. The proposed technique is used with MPE-FEC and AL-FEC therefore any modification of present implementation is not involved, because the raptor codes are already standardized for mobile WiMAX channel and all terminals. The proposed technique is useful by reducing the MPE-FEC and AL-FEC overhead, which may introduce extra delay. However, the delay can be adjusted by varying the AV content quality and, hence the burst size. 


\section{3. Brust Mapping for Downlink Mobile WiMAX}

To study the better throughput for orthogonal frequency division multiple access (OFDMA) based mobile WiMAX, the uplink and downlink burst mapping algorithm is given. The frequency band is divided into many subcarriers where radio resource is a frame. A frame is divided into two parts uplink and downlink subframes. In frequency division duplexing (FDD) these subframes are different frequency bands while in time division duplexing (TDD), subframes are time alternated. Resource allocation is performed by a slot which determines the throughput. The slot is done per burst with $\mathrm{AV}$ data of multiple or individual user. For downlink, the frame control header $(\mathrm{FCH})$ is used to map the burst profile of each allocation. As shown in Figure 8, each burst receive a rectangle allocation whose shape and position are specified through original information. To separate the redundant additional extra burst the unique connection identifier (CDI) is used. The burst size is rounded to a multiple of the height of the frame leading to resultant misuse of slots [15]. A treemap visualization algorithm [16] can be modified to meet the WiMAX requirements for efficient heuristic burst mappings. In this method, the bursts are assembled into vertical stripes by increasing the bursts width. The idea of treemap is basically to visualize the hard disk usage for the identification of large files. The tree structure of a file is represented by rectangles fitted into each other whose area is proportional to disk usage directories. The size of all directories divided into original rectangle in different slices whose width is proportional to the size of all directories [17]. So, this algorithm can be implemented for burst mapping by the aspect ratio of the bursts where ceiling operation have to be considered on frequency dimension and at symbol scale on time dimension. The sequential bursts are allocated through vertical stripes by varying its width. New additional bursts reduce the number of wasted slots which are added to stripe.

To know how the additional bursts are allocated to a stripe or slice, let consider a slice $\mathrm{S}$ composed of original bursts bí with $i=1, \ldots . n \mathrm{n}$, each burst requiring sí time slice. The height of the frame is denoted by $\mathrm{H}$. The temporary width of slice $\hat{\mathrm{w} S}$ is obtained as follows:

$\hat{\mathrm{W} S}=\frac{\sum \text { bíeS sí }}{\mathrm{H}}$

The temporary height hí of each burst is given by

Sí

$-\forall i=1, \ldots \ldots, n$ n

$\hat{W} S$

The height allocated to each burst is rounded to hí, where the sum of all rounded heights must be equal to the frame to match subchannel scale, where the operator $\ulcorner\cdot\lrcorner$ is used to the closest integer therefore the $\hat{h}^{\prime}$ also calculated as

$$
\text { hí = H }-\sum_{j=1}^{N \text { hí」 }-1} h j
$$

where $\forall i=1, \ldots \ldots, n$ в -1 ;

$$
i=n B
$$

The adaptive width of each burst ẅí is calculated as: sí

Ẅí $=\frac{}{\text { hí }} \quad \forall i=1, \ldots \ldots, n$ n

The width of stripe WS can be calculated as closest larger integer:

$$
\text { WS }=i=1, \ldots, n B \quad\ulcorner\ddot{\text { Wi }}\rfloor
$$

The number of wasted aditional or extra slots in stripe allocation can be calculated by $\mathrm{t} \omega$ as:

$$
€ \omega=(H . W S)-\sum_{i=1}^{n B} \text { sí }
$$

By computing $\hat{w} S$, the rounded height would not lead to any waste while there are still unallocated burst that make a new slice, sequentially add the extra bursts. If the slice is empty, the extra burst is directly added to next slice. If not, then pervious slot has to be done. By performing all computations if wasted slots are reduced by adding extra burst bí, which is defiantly added to the time slot or slice, and other bursts are then tried to be added.

For this calculation and burst mapping, the algorithm can be composed of a main while loop limited by the number nв of bursts. Then, for each time slot all allocated and unallocated bursts are tested to measure that which number is bounded by $\mathrm{n}$. The complexity $\mathrm{O}$ (n) of algorithm may be able to map the downlink bursts since there can be $n$ slots and for each one, the $n$ bursts can be tested. For the overflow of the wasted slots and additional bursts the scheduling algorithm is considered but it can not be implemented, where the bursts have to be dropped to maintain the highest throughput. Therefore, the scheduling of these bursts should be examined.

\section{PERFORMANCE ANALYSIS}

In order to assess the performance of IPTV over mobile WiMAX, a suite of relevant performance metrics are identified to properly target the system. Unmanaged VOD deployment is continually subjected to time varying bandwidth, packet delay and losses. 
Performance metrics observe packet transmissions, include packet loss, packet delay, packet jitter and traffic load throughput rates. Video playback quality is strongly related to packet loss and end-to-end packet delays. The following objective measures, which are widely used in video content performance analysis, are employed in this paper.

4. 1. Packet Loss Ratio Packet loss ratio (PLR) is the number of corrupted, dropped, or excessively delayed packets in relation to the total number of packets expected at the video client station. PLR can be calculated as follows [18]:

$\mathrm{PLR}=$ lost packets $/($ lost packets + received packets $)$

Another variation of this metric is the media loss rate (MLR) which track packet loss over time [19]: (Packets expected) - (Packets received)

MLR $=\frac{\text { Interval time in seconds }}{\text { In }}$

4. 2. Packet Delay Packet delay is the average packet transit time between the VoD server and the video client station. This metric can be calculated as follows [20]:

$$
\text { đ end }=\mathrm{Q}(\text { đ proc }+ \text { đ queue }+\mathrm{t} \text { trans }+ \text { đ prop })
$$

where $\mathrm{Q}$ is the number of network elements (routers, switches and firewalls) between the sender and receiver, đ proc is the processing delay at a given network element, d queue is the queuing delay at given network element, $t$ trans is the transmission time of a packet on a given link and d prop is the propagation delay across a given network link.

4. 3. Packet Jitter Packet jitter is defined as the variability in packet delay within a given media stream at the video client station. This metric can be calculated as:

$\mathrm{J}$ pkt $=\mathrm{t}$ actual $-\mathrm{t}$ expected

where $\mathrm{t}$ actual is the actual packet reception time and $\mathrm{t}$ expected is the expected packet reception time.

4. 4. Throughput Throughput is defined as the traffic load that the media stream will impress upon the network. It can be measured in bytes/sec (Bps) or bits/sec (bps). For CBR content, the throughput is constant and it can be calculated as:

Rmin $=($ frame size in bytes) (number of frames per second) / seconds

However, with variable bit rate encoders, the traffic loading is dynamic in nature and a function of the scene complexity and associated audio content. Consequently, variable bit rate (VBR) traffic loads are typically quoted in throughput ranges.
4. 5. Comparison between IPTV over Mobile WiMAX and over LTE To analyse the performance of robust IPTV over mobile WiMAX, a comparison between WiMAX and LTE is required. This study chose LTE over WiMAX as the technological foundation for its $3 \mathrm{G} / 4 \mathrm{G}$ wireless broadband network.

The throughput or peak network speed is an important factor to measure the performance. The peak network speed is usually quoted at layer 2 because of protocol overhead. LTE is a $4 \mathrm{G}$ wireless technology that Verizon Wireless and numerous leading wireless carriers have been chosen as their upgrade path beyond 3G technologies. Verizon Wireless will operate LTE in the $700 \mathrm{MHz}$ spectrum, which translates to unprecedented performance and data access. The given table shows the evolution of LTE.

TABLE 1. The evolution of GSM to LTE

\begin{tabular}{|c|c|c|c|c|}
\hline & 1xRTT & $\begin{array}{l}\text { 1Xev-do } \\
\text { Rel.0 }\end{array}$ & $\begin{array}{l}\text { 1Xev-DO } \\
\text { Rev.A }\end{array}$ & 3GPP LTE \\
\hline \multirow{2}{*}{ 离苛 } & $\begin{array}{l}153 \mathrm{Kbps} \\
\text { (downlink) }\end{array}$ & $\begin{array}{l}2.4 \mathrm{Mbps} \\
\text { (downlink) }\end{array}$ & $\begin{array}{l}\text { 3.1Mbps } \\
\text { (downlink) }\end{array}$ & $\begin{array}{l}\text { 100Mbps } \\
\text { (downlink) }\end{array}$ \\
\hline & $\begin{array}{l}153 \mathrm{Kbps} \\
\text { (uplink) }\end{array}$ & $\begin{array}{l}153 \mathrm{Kbps} \\
\text { (uplink). }\end{array}$ & $\begin{array}{l}1.8 \mathrm{Mbps} \\
\text { (uplink). }\end{array}$ & $\begin{array}{l}\text { 50Mbps } \\
\text { (uplink). }\end{array}$ \\
\hline \multirow{4}{*}{ 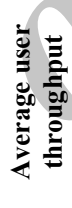 } & \multirow{4}{*}{$\begin{array}{l}60-80 \text { Kbps } \\
\text { (downlink) } \\
60-80 \text { Kbps } \\
\text { (uplink) }\end{array}$} & \multirow{2}{*}{$\begin{array}{l}400-700 \\
\text { Kbps } \\
\text { (downlink) }\end{array}$} & \multirow{2}{*}{$\begin{array}{l}600-1,400 \\
\text { Kbps } \\
\text { (downlink) }\end{array}$} & $5-12 \mathrm{Mbl}$ \\
\hline & & & & \\
\hline & & $60-80$ & $500-800$ & \multirow{2}{*}{$\begin{array}{l}\text { 2-5Mbps } \\
\text { (uplink) }\end{array}$} \\
\hline & & $\begin{array}{l}\text { Kbps } \\
\text { (uplink) }\end{array}$ & $\begin{array}{l}\text { Kbps } \\
\text { (uplink) }\end{array}$ & \\
\hline
\end{tabular}

TABLE 2. Technical difference between LTE and WiMAX

\begin{tabular}{|c|c|c|}
\hline & LTE & WiMAX 802.16E \\
\hline Technology & $\begin{array}{l}\text { MIMO Downlink: OFDM } \\
\text { Uplink:SC-FDMA }\end{array}$ & $\begin{array}{l}\text { MIMO Downlink: } \\
\text { OFDM Uplink: } \\
\text { FDMA }\end{array}$ \\
\hline Peak speeds & $\begin{array}{l}\text { Downlink: } 100 \text { Mbps ( } 20 \\
\text { MHz, 2x2 MIMO) } \\
\text { Uplink: } 50 \text { Mbps ( } 20 \\
\text { MHz, 1x2) }\end{array}$ & $\begin{array}{l}\text { Downlink: } 46 \mathrm{Mbps} \\
\text { Uplink: } 7 \mathrm{Mbps}\end{array}$ \\
\hline \multirow{2}{*}{$\begin{array}{l}\text { Average } \\
\text { user } \\
\text { throughput }\end{array}$} & $\begin{array}{l}5 \text { Mbps-12 Mbps } \\
\text { (downlink) }\end{array}$ & $\begin{array}{l}2 \text { Mbps-4 Mbps } \\
\text { (downlink) }\end{array}$ \\
\hline & 2 Mbps-5 Mbps (uplink). & $\begin{array}{l}500 \mathrm{Kbps}-1.5 \mathrm{Mbps} \\
\text { (uplink). }\end{array}$ \\
\hline $\begin{array}{l}\text { One-way } \\
\text { airlink } \\
\text { latency }\end{array}$ & $15 \mathrm{~ms}$ & $50 \mathrm{~ms}$ \\
\hline Bandwidth & $\begin{array}{l}20 \mathrm{MHz}, 15 \mathrm{MHz}, 10 \\
\mathrm{MHz}, 5 \mathrm{MHz} \text {, and }<5 \\
\mathrm{MHz} .\end{array}$ & $\begin{array}{l}3.5 \mathrm{MHz}, 5 \mathrm{MHz}, 7 \\
\mathrm{MHz}, 8.75 \mathrm{MHz}, 10 \\
\mathrm{MHz} .\end{array}$ \\
\hline Spectrum & $\begin{array}{l}\text { Verizon Wireless will use } \\
700 \mathrm{MHz} \text {, but LTE can be } \\
\text { deployed in various } \\
\text { frequencies. Use of the } \\
700 \mathrm{MHz} \text { frequency helps } \\
\text { to increase in-building } \\
\text { coverage for wireless } \\
\text { signals. }\end{array}$ & $2.3,2.5,3.5,5.8 \mathrm{GHz}$ \\
\hline Mobility & $\begin{array}{l}\text { Targeted mobility up to } \\
350 \mathrm{kmph} \text {. }\end{array}$ & $\begin{array}{l}\text { Targeted mobility up } \\
\text { to } 120 \mathrm{kmph} \text {. }\end{array}$ \\
\hline
\end{tabular}


LTE and WiMAX are able to provide wide range coverage, high data rates, secured transmission and mobility supported at vehicular speeds. The quality of service (QoS) is required to transmit audio, video, voice, data services such as video gaming and mobile IPTV. The 3GPP LTE is the latest standard in the cellular network technology that previously realized the GSM/EDGE, UMTS/HSxPA network technologies of mobile subscribers. A radio access of LTE is named Evolved UMTS Terrestrial Radio Access Network (EUTRAN) which improves end-user throughputs, sector capacity and reduces user plane latency to support IPTV services, with full mobility. For carrying all types of traffics, LTE is planned to provide IP-based traffic with end-to-end Quality of service (QoS).

TABLE 3. Techno-economic differences between WiMAX \& LTE [21]

\begin{tabular}{|c|c|c|}
\hline & WiMAX & LTE \\
\hline Foundation & Based on IP & $\begin{array}{l}3 \mathrm{G} \text { an evolution from a } \\
\text { voice traffic design }\end{array}$ \\
\hline Deployment & $\begin{array}{l}\text { Yet to deploy in } \\
\text { volume (2009) } \\
\text { Deployed recently }\end{array}$ & $\begin{array}{l}3 \mathrm{G} \text { has a substantial base } \\
\text { station population ( } 128 \\
\text { commercial HSPA } \\
\text { networks rolled out and } \\
\text { over } 300 \text { HSPA devices) }\end{array}$ \\
\hline Maturity & $\begin{array}{l}\text { Mobile WiMAX } \\
\text { under ratification } \\
\text { Mobile WiMAX } \\
\text { has to win a } \\
\text { customer base }\end{array}$ & $\begin{array}{l}3 \mathrm{G} \text { technology is maturing } \\
3 \mathrm{G} \text { customer base is } \\
\text { established by evolution } \\
\text { from GSM }\end{array}$ \\
\hline User devices & $\begin{array}{l}\text { WiMAX will be } \\
\text { part of existing } \\
\text { hardware such as } \\
\text { laptops and PDAs }\end{array}$ & $\begin{array}{l}3 \mathrm{G} \text { uses dedicated } \\
\text { hardware or plug in cards }\end{array}$ \\
\hline Equipment cost & $\begin{array}{l}\text { Owing to standards, } \\
\text { cheaper }\end{array}$ & $\begin{array}{l}\text { No standards available as } \\
\text { on date, hence may be } \\
\text { costlier (2009) }\end{array}$ \\
\hline $\begin{array}{l}\text { Deployment } \\
\text { cost }\end{array}$ & Higher & Lower \\
\hline
\end{tabular}

TABLE 4. Similarities between LTE \& WiMAX [21]

\begin{tabular}{ll}
\hline \multirow{2}{*}{ OFDMA } & $\begin{array}{l}\text { OFDMA supports advanced antenna technologies, } \\
\text { such as MIMO, STC, and beamforming OFDMA is } \\
\text { adopted as the basis of mobile WiMAX } \\
\text { 3GPPP's Long Term Evolution (LTE) project plans to } \\
\text { incorporate OFDMA7 }\end{array}$ \\
LTE is moving from a circuit-switched to all IP \\
IP is built into mobile WiMAX based on the IEEE \\
802.16 air interface standard
\end{tabular}

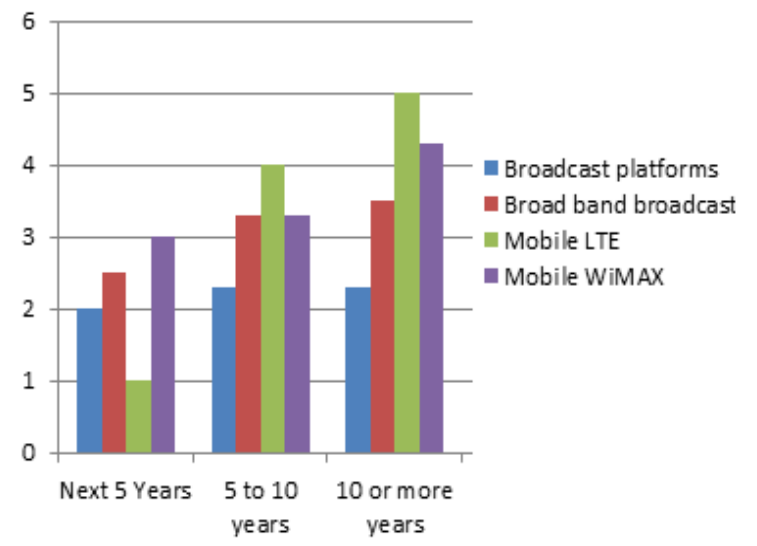

Figure 9. Technologies expected to complement TV broadcasting in the futur

LTE core network is the Evolved Packet Core (EPC) which allows for connections and hand-over to other fixed line and wireless access to deliver a seamless mobility experience. LTE minimizes the system and UE complexities and enable co-existence with other 3GPP Radio Access Technologies (RATs). Using evolved multimedia broadcast, multicast service (E-MBMS) 3 GPP provides the IPTV services multi-cell synchronized transmission technology. In the viability of the LTE standard and its future potential $4 \mathrm{G}$ technologies determine the best fit for its network. IPTV delivery over WiMAX or LTE both have same issues as number of channels depends on viewer's waiting time (start up delay) and it does not depend on number of requests. A quality of transmission channel decreases due to a noisy environment. The symbol error rate (SER) will increase with respect to proliferation of fading frequency.

The overall radio performance is slightly equal with LTE faintly overtaking WiMAX. Strategic considerations on the future of TV broadcasting especially IPTV is shown in Figure 9 which gives an overview of the complementary technologies that are expected to emerge over the next 10 years and beyond. The height of the bars corresponds to the number of responses [22].

It can be seen that, terrestrial distribution will remain a significant delivery platform but will be complemented by mobile (LTE) networks. In the next five years the introduction of DVB-T2 alongside DVB$\mathrm{T}$ is expected. There will still be a minimal use of nonbroadcast technologies (mobile networks) for linear services. Furthermore, IPTV is expected to grow rapidly. Over the next 5-10 years, LTE will be available, but its capacity may be insufficient for significant delivery of linear services. Hence, it is expected that hybrid solutions will be adopted which will employ the terrestrial platform as one delivery mechanism. Clearly, there will still be an increasing importance for IP-based distribution over the internet. 


\section{CONCLUSION}

In this work, an analysis of the effectiveness of AL-FEC and MPE-FEC schemes are discussed for IPTV delivery over mobile WiMAX and a technique is proposed to add an extra burst to resolve the issues. The mobile WiMAX downlink burst mapping algorithm is also discussed for the measurements of flooding of redundant slots. Based on the performed analysis, an optimization of the quality assessment is presented which analyses the adapted rateless channel coding. It is also described that how to transmit mobile IPTV service over cellular system specially, over LTE network. The delay time and packet loss rate are critical parameters for mobile IPTV service with highly QoS. IPTV has witnessed a rapid evolution moved from the STB to the phone, the PC and soon the game console. This paper reflects the status of techniques that are already deployed and researched to improve the IPTV issues. While IPTV of today still provides the lean back experience, where new researches are required, the future of IPTV is social and mobile and this future is already apparent.

\section{REFERENCES}

1. Kim, B., Choi, J., Kim, J. and Shin, Y., "A study on frame encryption for protecting IPTV contents", in Advanced Communication Technology (ICACT), 13th International Conference on, IEEE., (2011), 1484-1488.

2. Park, W. K., Choi, C. S., Jeong, Y. K. and Han, I., "An implementation of the broadband home gateway supporting multi-channel IPTV service", in Consumer Electronics, ISCE'06. 2006 IEEE Tenth International Symposium on, IEEE, (2006), 15

3. Ahmad, S., Hamzaoui, R. and Al-Akaidi, M., "Video multicast using unequal error protection with Luby Transform codes", (2009).

4. MacKay, D. J. C., "Fountain codes", in Communications, IEE Proceedings-, IET. Vol. 152, (2005), 1062-1068.

5. Schreier, R. M., Rahman, A. M. T. I., Krishnamurthy, G. and Rothermel, A., "Architecture analysis for low-delay video coding", in Multimedia and Expo, 2006 IEEE International Conference on, IEEE, (2006), 2053-2056.

6. Al-Jobouri, L., Fleury, M. and Ghanbari, M., "Robust IPTV delivery with adaptive rateless coding over a mobile WiMAX channel", ISRN Communications and Networking, Vol. 2011, No., (2011), 24.

7. Jain, R., So-In, C. and Al Tamimi, A., "System-level modeling of IEEE 802.16 E mobile WiMAX networks: key issues", Wireless Communications, IEEE, Vol. 15, No. 5, (2008), 73 79.

8. Ahmed, T., Mehaoua, A., Boutaba, R. and Iraqi, Y., "Adaptive packet video streaming over IP networks: a cross-layer approach", Selected Areas in Communications, IEEE Journal on, Vol. 23, No. 2, (2005), 385-401.

9. Vassiliou, V. and Zinonos, Z., "An analysis of the handover latency components in mobile ipv6", Journal of Internet Engineering, Vol. 3, No. 1, (2010).

10. Pinola, J. and Pentikousis, K., "IPTV over WiMAX with MIPv6 handovers", in Vehicular Technology Conference, 2009. VTC Spring 2009. IEEE 69th, IEEE, (2009), 1-5.

11. Lee, H. B., Han, Y. H. and Min, S. G., "Network Mobility Support Scheme on PMIPv6 Networks", International Journal of Computer Networks \& Communications (IJCNC), Vol. 2, No. 5, (2010).

12. Yang, X., "Handover in DVB-H: investigations and analysis, Springer, (2008).

13. Luo, F. L., "Mobile Multimedia Broadcasting Standards: Technology and Practice, Springer, (2008).

14. Penttinen, J., Jolma, P., Aaltonen, E. and Väre, J., "The DVB-H Handbook: The Functioning and Planning of Mobile TV, Wiley, (2010).

15. Ohseki, T., Morita, M. and Inoue, T., "Burst construction and packet mapping scheme for OFDMA downlinks in IEEE 802.16 systems", in Global Telecommunications Conference, 2007. GLOBECOM'07. IEEE, IEEE, (2007), 4307-4311.

16. Bruls, M., Huizing, K. and Van Wijk, J. J., "Squarified treemaps", in Data Visualization, Citeseer, (2000), 33-42.

17. Shneiderman, B., "Tree visualization with tree-maps: 2-d spacefilling approach", ACM Transactions on graphics (TOG), Vol. 11, No. 1, (1992), 92-99.

18. Almomani, O., Hassan, S. and Nor, S., "Effects of packet size on FEC performance", Proc. NetApps2008, Malaysia, (2008), 1-4.

19. Krejci, J., "MDI measurement in the IPTV", in Systems, Signals and Image Processing, 2008. IWSSIP 2008. 15th International Conference on, IEEE, (2008), 49-52.

20. Kurose, J. and Ross, K., "Computer Networks: A Top Down Approach Featuring the Internet, Pearson Addison Wesley, (2006).

21. Raut, S., "WiMAX or LTE: Which Technology to Adopt? A Comprehensive Comparative Study"', Communications of the IBIMA, Vol. 9, (2009).

22. Beutler, R., "The digital dividend of terrestrial broadcasting", Springer, (2011). 


\title{
Analysis of Packet Loss and Latency Control for Robust IPTV over Mobile WiMAX and LTE Assessment

\author{
M. Akram a, F. Zafar b \\ a Department of Information Technology, University of Central Punjab, Lahore, Pakistan \\ ${ }^{b}$ Department of Computer Science, Government College University, Lahore, Pakistan
}

Paper history:

Received 7 February 2012

Received in revised form 5 September 2012

Accepted 15 November 2012

Keywords:

IPTV

WiMAX

LTE

IPv6

MPEG

FEC

Coding

Streaming

Signal Brust

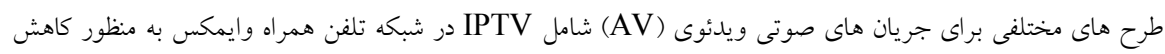

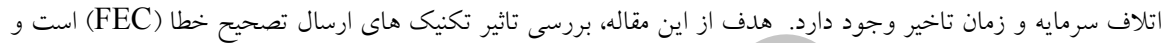

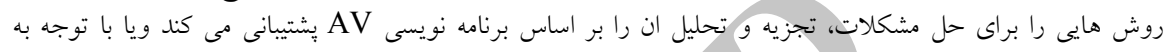

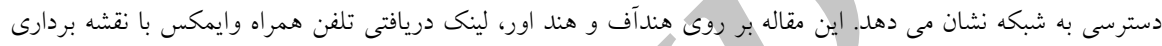

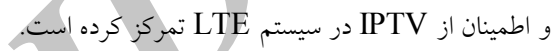




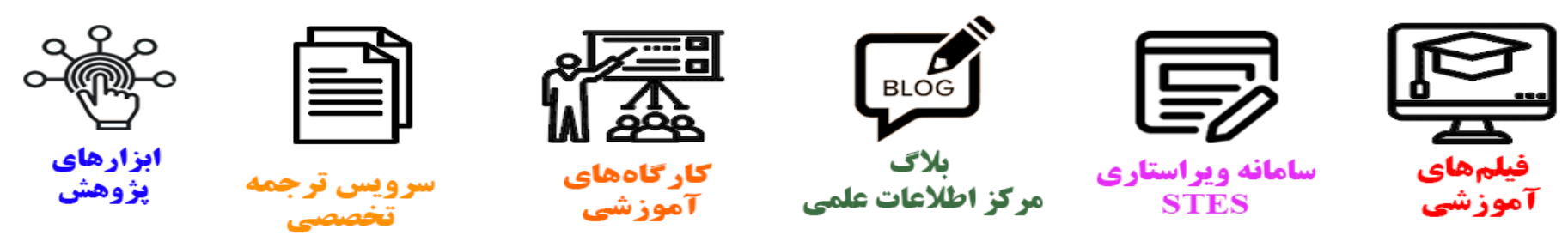

\section{(c)}

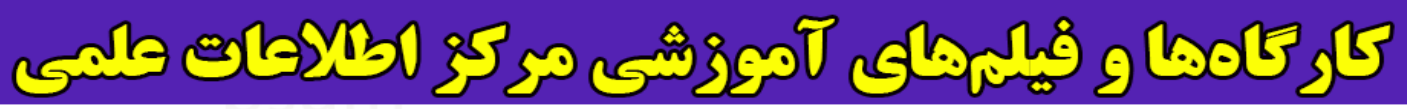
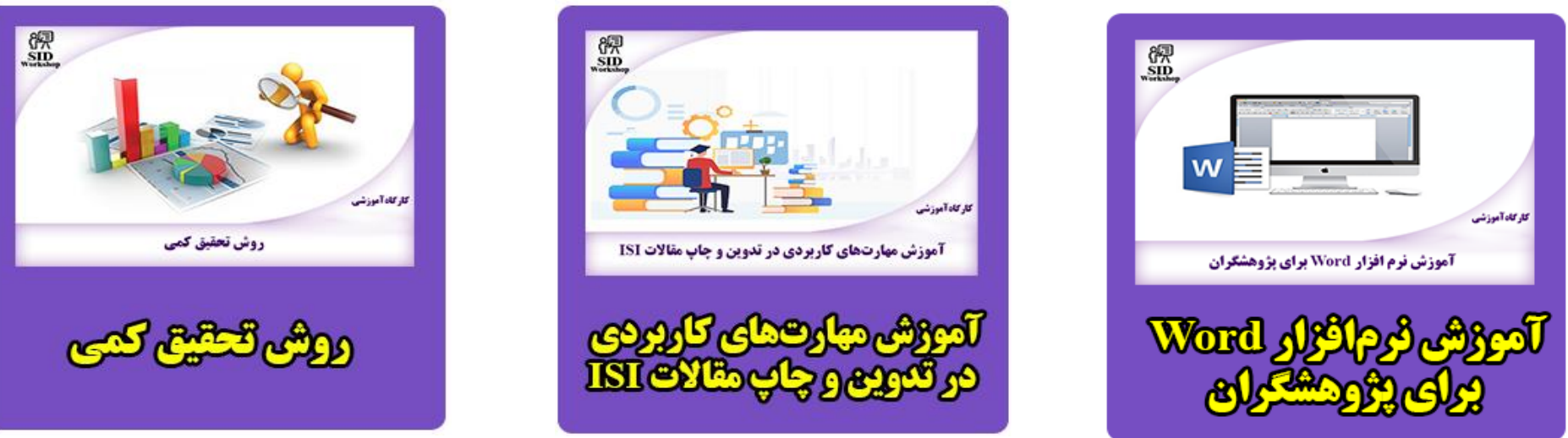